\title{
Coordinate Pulsatile Insulin Secretion by Chronic Intraportally Transplanted Islets in the Isolated Perfused Rat Liver
}

\author{
Niels Porksen, ${ }^{*}$ Stephen Munn, ${ }^{\ddagger}$ Deanna Ferguson, ${ }^{\star}$ Timothy O’Brien, ${ }^{*}$ Johannes Veldhuis, ${ }^{5}$ and Peter Butter* \\ *Endocrine Research Unit and ${ }^{\ddagger}$ Department of Transplant Surgery, Mayo Clinic, Rochester, Minnesota 55905; \\ and ${ }^{8}$ Department of Medicine, University of Virginia, Charlottesville, Virginia 22908
}

\begin{abstract}
In the present studies we sought to address the following questions: do chronically transplanted intrahepatic islets (IHI-Tx) secrete insulin in a coordinate pulsatile manner, and, if so, is reestablishment of this coordinate pulsatility a function of time after transplantation? We studied isolated perfused livers at $10 \mathrm{mM}$ glucose from 27 rats rendered diabetic with streptozotocin and then transplanted with $\sim 2$ $\times 10^{3}$ islets, $2(n=5), 7(n=5), 30(n=5)$, and $200(n$ $=12$ ) d after transplantation. 12 out of 12 of the 200-d IHITx secreted insulin in coordinate pulses (frequency $3.9 \pm 0.3$ pulses/h, amplitude $15.2 \pm 2.4 \mathrm{nmol} / \mathrm{min}$ ). In contrast, one out of five 2-d, zero out of five 7-d, and one out of five 30d IHI-Tx showed pulsatile insulin secretion. Insulin secretion was markedly greater $(76 \pm 13$ vs $13 \pm 3 \mathrm{nmol} / \mathrm{min}, P$ $<0.0001$ ) in the 200-d versus early IHI-Tx. Pentobarbital $25 \mu \mathrm{g} / \mathrm{ml}$ had no effect on total (13.9 $\pm 3.9 \mathrm{vs} 15.9 \pm 3.9 \mathrm{nmol} /$ min), nonpulsatile $(12.9 \pm 3.5 \mathrm{vs} 14.1 \pm 3.3 \mathrm{nmol} / \mathrm{min})$, or pulsatile (pulse amplitude $17.6 \pm 4.5 \mathrm{vs} 20.0 \pm 4.2 \mathrm{nmol} / \mathrm{min}$, pulse frequency $4.1 \pm 0.3$ vs $4.0 \pm 0.7 \mathrm{pulses} / \mathrm{h}$ ) insulin secretion. Using synaptophysin, islet innervation was documented in 12 out of 12 200-d IHI-Tx but in none of the early IHITx. We conclude that established ( $\sim 200$ d) IHI-Tx secrete insulin in a coordinate pulsatile manner and that establishment of coordinate pulsatile insulin secretion by IHI-Tx is accompanied by increased total insulin secretion and is associated with islet reinnervation. (J. Clin. Invest. 1994. 94: 219-227.) Key words: islet transplantation • insulin • pulsatility • islet innervation - diabetes mellitus
\end{abstract}

\section{Introduction}

Intraportal transplantation of pancreatic islets is a therapeutic option under active investigation for patients with insulin-dependent diabetes mellitus (1-4). Intrahepatic autotransplantation of pancreatic islets salvaged after pancreatectomy for pancreatitis has achieved sustained insulin secretion and near normal glycemic control in humans (5), although a large number of islets appears to be critical for success. Shorter periods of insulin secretion and glycemic control have been obtained in

Address correspondence to Peter C. Butler, M.D., Endocrine Research Unit, 5-164 West Joseph, Mayo Clinic, Rochester, MN 55905.

This work was presented in part at the National Meeting of the American Federation of Clinical Research in Washington, DC on 30 April 1993.

Received for publication 29 December 1993 and in revised form 14 March 1994.

The Journal of Clinical Investigation, Inc.

Volume 94, July 1994, 219-227 patients with insulin-dependent diabetes mellitus transplanted with islets $(3,4)$. Relatively little is yet known about the regulation of insulin secretion in intrahepatic transplanted islets.

In health it is well known that plasma insulin concentrations oscillate with both a high (6) and low periodicity ( 12 vs $60-$ $90 \mathrm{~min}$ ) (7). The low frequency oscillations most likely reflect feedback between insulin secretion and hepatic glucose release since they can be entrained by perturbations in circulating glucose concentration (8), and therefore it is not surprising that low frequency oscillations are retained in dogs with intrahepatic transplanted islets (9). In contrast, high frequency oscillations appear to be due to coordinate insulin secretory bursts by islets dispersed throughout the exocrine pancreas and are retained in the isolated perfused pancreas during a constant glucose infusion (10). The latter suggests that the pacemaker responsible for high frequency pulses is intrapancreatic and that the islets dispersed within the pancreas communicate most likely by an intrinsic neural network analogous to the Purkinje system in the heart.

It is unknown if intrahepatic transplanted islets secrete insulin in a high frequency pulsatile manner. If coordinate insulin secretion is mediated by the intrapancreatic neural network (11), it might be expected that the dispersal of transplanted islets throughout the liver would result in loss of pulsatile insulin secretion. However, since intrahepatic transplanted islets are reinnervated (12-14), a process that has been identified in occasional islets $\sim 4 \mathrm{wk}$ after transplantation (12) and has become clearly established by 14 wk (14), it is plausible that coordinate pulsatile insulin secretion is restored in chronically transplanted islets.

This study was undertaken to determine if transplanted intrahepatic islets secrete insulin in a coordinate high frequency pulsatile manner. In view of the potential importance of duration of transplantation on this process, we performed the studies 2 , 7,30 , and $200 \mathrm{~d}$ after intraportal transplantation of islets in streptozotocin-induced diabetic rats. To avoid the potential confounding variables of circulating insulin secretagogues, we used an open-loop isolated liver perfusion system to detect the presence of coordinate insulin secretory bursts by the transplanted islets.

In vivo anesthetic doses of barbiturates have been shown to acutely inhibit insulin secretion $(15,16)$. Anesthetic doses of barbiturates have marked suppressive effects on spontaneous activity in the central nervous system (17) but relatively minor effects on intraorgan spontaneous neural activity such as occurs in the heart (18). Therefore, if pacemaker activity and interislet communication are achieved by a system analogous to the sinoatrial node/Purkinje tissue system, barbiturates at an anesthetic dose would be expected to have, at most, a minor impact on pulsatile insulin secretion. Therefore, after preliminary data indicated that chronic intrahepatic islet transplants secreted insulin in a pulsatile manner, we sought to determine the effect of an 
Table I. Characteristics of Rats Included in Study

\begin{tabular}{|c|c|c|c|c|c|c|}
\hline Days $T x$ & $\begin{array}{c}\text { Number } \\
\text { of rats }\end{array}$ & $\begin{array}{l}\text { Weight at } \\
\text { study* }\end{array}$ & $\begin{array}{l}\text { Glucose before } \\
\text { transplantation }\end{array}$ & $\begin{array}{c}\text { Islets } \\
\text { transplanted }\end{array}$ & $\begin{array}{l}\text { Glucose after } \\
\text { transplantation }\end{array}$ & $\begin{array}{l}\text { Glucose on } \\
\text { study day }\end{array}$ \\
\hline & & $g$ & $m M$ & number & $m M$ & $m M$ \\
\hline 2 & 5 & $227 \pm 9$ & $19.4 \pm 0.6$ & $2040 \pm 65$ & $4.6 \pm 0.2$ & $4.6 \pm 0.2$ \\
\hline 7 & 5 & $242 \pm 14$ & $18.3 \pm 0.7$ & $2035 \pm 26$ & $4.9 \pm 0.2$ & $5.0 \pm 0.2$ \\
\hline 30 & 5 & $303 \pm 3$ & $21.3 \pm 0.7^{\| \prime}$ & $2056 \pm 29$ & $4.6 \pm 0.1$ & $4.2 \pm 0.2$ \\
\hline 200 & 12 & $441 \pm 5$ & $19.2 \pm 0.4$ & $2128 \pm 44$ & $4.2 \pm 0.3$ & $5.3 \pm 0.3^{1}$ \\
\hline
\end{tabular}

* Increase with time $(P<0.02, r=0.48), \quad{ }^{\ddagger}$ Tested by regression analysis for change over time. $\quad \| 7<30(P<0.05), \quad 130<200(P$ $<0.05),{ }^{\&}$ Tested by Newman-Keul's test for multiple comparisons.

anesthetic dose of pentobarbital on pulsatile insulin secretion by intrahepatic islets.

\section{Methods}

\section{Transplantation}

Inbred Fisher (F344) rats, age 11-13 wk were used as donors as well as recipients for islet transplantation as described previously (19). A total of 29 rats was rendered diabetic with streptozotocin $(1 \mathrm{mg} / 30 \mathrm{~g})$. After $7 \mathrm{~d}$, isograft transplantation was performed using $2.0 \pm 0.1 \times 1,000$ islets. Donor rats were killed using ether, and the pancreas was immediately cannulated and injected with type XI collagenase (Sigma Immunochemicals, St. Louis, MO) in Hanks' solution. Slowly shaken digestion was carried out at $37^{\circ} \mathrm{C}$ for $18 \mathrm{~min}$. The digested pancreas was screened and subjected to density gradient centrifugation using dextran (Sigma Immunochemicals). Then, islets were handpicked for further purification and enumeration under a dissecting microscope. Transplantation of islets was performed during ether anesthesia. The abdomen was opened using a midline incision, and islets were injected into a tributary of the portal vein using a $1-\mathrm{cm}^{3}$ insulin syringe with a 25 -gauge needle. The abdomen was closed using 3-0 coated vicryl. Blood glucose concentrations were monitored by sampling blood from a tail vein and were measured by the glucose oxidase method (Accu-check-III; Boehringer Mannheim Corp., Indianapolis, IN). By the second postoperative day, blood glucose concentrations had returned to normal in all 29 rats and remained so throughout the study (Table I). After transplantation, rats were kept in boxes, two rats per box, and were allowed free rat chow (LaboChow; Purina Mills, St. Louis, MO). Of the original 29 diabetic rats, 27 were included in the present studies, and 2 were excluded because of hepatic ischemia during the isolated hepatic perfusion.

\section{Liver isolation}

Rats were studied at either $2(n=5), 7(n=5), 30(n=5)$, or 200 $(n=12) \mathrm{d}$ after transplantation (Table I) using the isolated perfused liver. No food was allowed after 5 o'clock the evening before the study. After induction of ether anesthesia, the abdomen was opened using a transverse incision. The liver was dissected free from capsule and hepatic ligament. Silk sutures were tied loosely around the portal vein using two proximal sutures and one distal suture. One silk suture was tied loosely around the inferior cava vein. The bile duct was isolated and dissected to allow free flow of bile. The distal portal suture was tied, and a 16-gauge catheter (Insyte; Becton Dickinson, Sandy, UT) was inserted into the portal vein. The two proximal sutures were tied securing the catheter at position with the catheter tip distal to the branching of the portal vein and ligating the hepatic artery. Then, the liver was perfused (avoiding air bubbles) with oxygenated Krebs buffer, $\mathrm{pH} 7.4$, glucose $10 \mathrm{mmol} /$ liter, partial pressure of oxygen $\sim 490 \mathrm{mmHg}$, partial pressure of carbon dioxide $\sim 8 \mathrm{mmHg}$, free $\mathrm{Ca}^{2+} 1.2 \mathrm{mmol} /$ liter, and $0.25 \%$ BSA (Armour Pharmaceutical Co., Kankakee, IL). The inferior cava suture was tied to prevent reverse flow into the liver via the hepatic vein. On perfusion, an instantaneous and homogeneous pallor of the entire liver indicated successful perfusion. Thereafter, the chest was opened, the inferior cava was dissected and opened, and a polyethylene catheter (i.d. $1.67 \mathrm{~mm}$, o.d. $2.42 \mathrm{~mm}$ ) (Intramedic; Clay Adams, Parsippany, NJ) was inserted and secured with two sutures tied proximal to the liver. The liver was then removed from the rat by careful dissection from the diaphragm and capsule. The liver was weighed and then connected to the perfusion apparatus.

\section{Perfusion}

An open-loop perfusion system was used. The liver was placed on soft rubber to avoid pressure, placed in a humidified heated $\left(37^{\circ} \mathrm{C}\right)$ closed chamber, and wrapped in saline-moistened gauze. Krebs buffer was pumped from a perfusion reservoir to an oxygenator $\left(95 \% \mathrm{O}_{2}, 5 \% \mathrm{CO}_{2}\right)$ (20) by a peristalsis pump (Manostat, Varistaltic S; Baxter Scientific, McGraw Park, IL). Excess oxygen and buffer were recirculated to the buffer reservoir by a sialastic tube, thus leading to oxygenation of reservoir buffer. From the lower bulb, buffer was delivered to the liver at a fixed pressure $\left(\sim 20 \mathrm{~cm} \mathrm{H}_{2} \mathrm{O}\right)$. Buffer was sampled for gas analyses every $15 \mathrm{~min}$ from the hepatic perfusate and effluent. Effluent was collected every minute from the hepatic catheter, and volume was measured to obtain flow rate per minute. At all times, care was taken to avoid traction and/or pressure on portal and hepatic catheters. The liver was weighed at the end of the study.

Hepatic viability was assessed by conventional methods $(21,22)$ including $(a)$ gross appearance, $(b)$ oxygen uptake, potassium release, and $\mathrm{pH},(c)$ flow rates, and $(d)$ release of liver enzymes (Table II). At the end of each experiment, several slices of liver were sectioned and fixed by $10 \%$ neutral buffered formalin for $4 \mathrm{~h}$ before being transferred to $70 \%$ ethanol and routinely processed for paraffin embedding. Serial sections, 4- $\mu \mathrm{m}$ thick, were mounted on chromalum-coated glass slides, deparaffinized, and stained with hematoxylin and eosin, and the peroxidase-antiperoxidase method for insulin (guinea pig antiporcine; Dako Corp., Carpinteria, CA) (1/1,000), IAPP (rabbit anti-human; Penin-

Table II. Data Demonstrating Viability of Perfused Livers

\begin{tabular}{|c|c|c|c|c|}
\hline Days Tx & $\begin{array}{l}\text { Number } \\
\text { of rats }\end{array}$ & $\begin{array}{l}\text { Oxygen } \\
\text { uptake* }\end{array}$ & LDH $^{*}$ & Flow* \\
\hline & & $\underset{\text { min }}{\text { mmolg per }}$ & Uniter & $\begin{array}{l}\text { ml/min per } \\
\text { gram }\end{array}$ \\
\hline 2 & 5 & $0.80 \pm 0.10$ & $2.8 \pm 0.5$ & $2.62 \pm 0.36$ \\
\hline 7 & 5 & $0.73 \pm 0.07$ & $8.9 \pm 3.7$ & $2.26 \pm 0.12$ \\
\hline 30 & 5 & $0.87 \pm 0.03$ & $5.8 \pm 1.3$ & $2.16 \pm 0.11$ \\
\hline 200 & 12 & $0.96 \pm 0.10$ & $27 \pm 19$ & $2.18 \pm 0.15$ \\
\hline
\end{tabular}

* Tested by Newman-Keul's test for multiple comparisons. LDH, lactate dehydrogenase. 


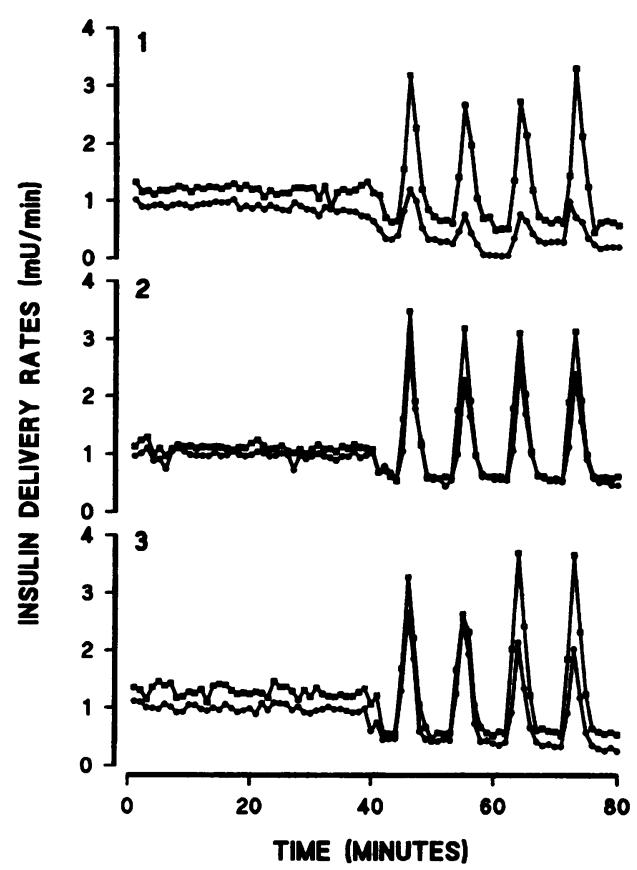

Figure 1. Insulin delivery rate (milliUnits per minute) to (black boxes) and from (black circles) control isolated perfused rat livers during a constant (0-40 $\mathrm{min})$ versus pulsatile $(41-80 \mathrm{~min})$ insulin infusion of $1.0 \mathrm{mU} / \mathrm{min}$.

sula Laboratories, Inc., Belmont, CA) $(1 / 1,000)$, and synaptophysin (mouse anti-human; ICN, Costa Mesa, CA) (1/40).

\section{Protocols}

Protocol 1: controls. To control for assay variability, pooled buffer was assayed in replicate ( $n=3$ sample replicates) in the same manner as study samples. To control for model variability, constant and pulsatile insulin were delivered to isolated perfused livers $(n=3)$ with no transplanted islets. Human insulin (Humulin; Eli Lilly \& Co., Indianapolis, IN) was infused upstream to the portal vein catheter. A mixing coil consisting of $50 \mathrm{~cm}$ of coiled plastic tubing was interposed between the site of infusion of insulin and the liver to assure proper mixing of insulin and buffer before the liver. Samples of both perfusate (after the mixing coil) and effluent were collected at 1-min intervals. Insulin was infused at a constant rate of $1 \mathrm{mU} / \mathrm{min}$ for $\mathbf{4 0} \mathrm{min}$ and then in a pulsatile manner for $40 \mathrm{~min}$. During pulsatile delivery, the total insulin delivery rate remained unchanged $(1.0 \mathrm{mU} / \mathrm{min})$, but the basal rate was decreased to $0.5 \mathrm{mU} / \mathrm{min}$, and at $10-\mathrm{min}$ intervals the rate was increased to 3.5 , 2 , and $1 \mathrm{mU} / \mathrm{min}$ for $1 \mathrm{~min}$ each.

Protocol 2: studies. The pattern of insulin secreted by hepatic transplanted islets and its relationship to duration of transplantation were measured by the isolated perfused liver technique described above in rats $2(n=5), 7(n=5), 30(n=5)$, and $200(n=12) \mathrm{d}$ after islet transplantation (Tx $).^{1}$ After $15 \mathrm{~min}$ of perfusion with buffer containing glucose at a concentration of $10 \mathrm{mM}$, perfusate effluent was sampled at 1-min intervals for $40 \mathrm{~min}$. The 15 -min run in period ensured that a first-phase response to hyperglycemia would not be detected as a pulse.

To measure first-phase insulin secretion in 3 of the 12 200-d Tx rats and all 2-, 7-, and 30-d Tx rats, a 50- $\mu$ g bolus of glucagon was given over $30 \mathrm{~s}$ at $\mathrm{t}=41 \mathrm{~min}$, and sampling continued for an additional $5 \mathrm{~min}$.

Having established in the first eight 200-d transplanted livers that insulin secretion was pulsatile, we sought to determine if an anesthetic

1. Abbreviation used in this paper: $\mathrm{Tx}$, transplantation.

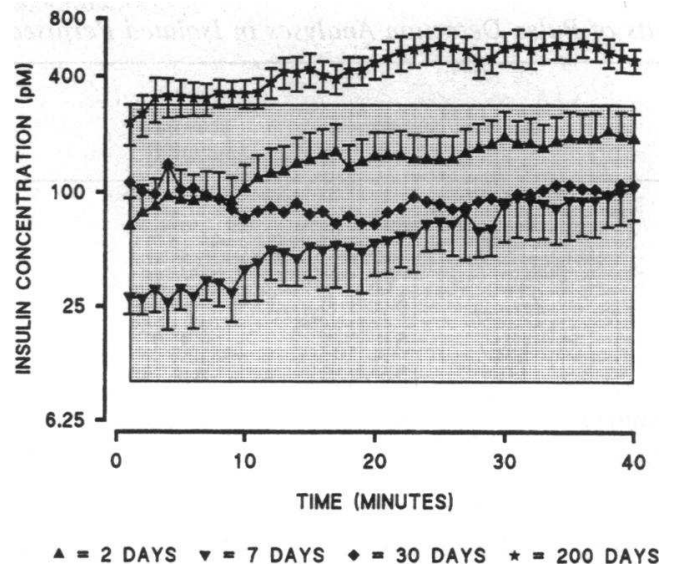

Figure 2. The mean insulin concentrations for the 2-, 7-, 30-, and 200d Tx groups are shown throughout the $10 \mathrm{mM}$ glucose perfusion. The range of the insulin assay is shown by the shaded area (logarithmic scale as per assay). Most 200-d Tx samples had to be diluted for assay. SEM bars are omitted from some points for clarity because of extensive overlap.

concentration of pentobarbital $(25 \mu \mathrm{g} / \mathrm{ml})$ perturbed the coordinate pulsatile insulin secretion. Therefore, in four of the 200-d Tx rats, the glucagon bolus was omitted, and a constant infusion of pentobarbital (Sigma Immunochemicals) $25 \mu \mathrm{g} / \mathrm{ml}$ was administered via the perfusate (glucose concentration remaining $10 \mathrm{mM}$ ) from $t=41-70 \mathrm{~min}$.

Measurements. All buffer samples were placed on ice immediately and frozen at $-20^{\circ} \mathrm{C}$ after each study. C-peptide and insulin were assayed in replicates by radioimmunoassay. Radioimmunoassay for insulin was carried out in $0.05 \mathrm{M}$ phosphate-buffered saline with $0.025 \mathrm{M}$ EDTA, $1 \%$ BSA, and $0.01 \%$ sodium azide. Standards and unknowns were preincubated for $3 \mathrm{~h}$ with guinea pig antiinsulin antibody (1013 [cross-reactivity rat I insulin 100\%, rat II insulin 70\%]; Linco Research Inc., St. Louis, MO) specific for rat insulin and then were incubated overnight after addition of ${ }^{125} \mathrm{I}$-insulin tracer $(10,000 \mathrm{cpm} /$ tube $)$. Separation of bound insulin was accomplished with goat anti-guinea pig gamma globulin in the presence of $1 \%$ polyethylene glycol during a 2$h$ incubation. After centrifugation, the free portion was discarded, and the bound portion (pellet) was counted on a gamma counter. The counts per minute of each tube were calculated as a percentage of maximum binding (that bound in the absence of cold antigen), and unknowns were read from a standard curve. The assay sensitivity and range were $75-2,500 \mathrm{pg} / \mathrm{ml}$ using a $100-\mu l$ sample size. Samples with insulin concentrations greater than assay range were diluted to bring them within the linear portion of the binding curve. Intraassay variation was estimated by measuring the insulin concentration from a pooled sample of effluent 40 times at concentrations corresponding to low, middle, and high binding on the insulin assay binding curve. The intra- and interassay variation for insulin was 10.7 (low), 7.0 (medium), 10.1 (high), and 7.5 (low), 7.7 (medium), and $4.1 \%$ (high), respectively. Rat C-peptide was also measured by RIA using a commercial kit (Linco Research Inc.). 100 $\mu \mathrm{l}$ of antibody was added to $100 \mu \mathrm{l}$ of standard or sample and incubated at $4^{\circ} \mathrm{C}$ overnight. Then, $100 \mu \mathrm{l}$ of ${ }^{125} \mathrm{I}$-labeled rat $\mathrm{C}$-peptide $(15,000$ $\mathrm{cpm} /$ tube) was added for an additional $24-\mathrm{h} 4^{\circ} \mathrm{C}$ incubation. Thereafter, separation of bound C-peptide was accomplished by the addition of anti-guinea pig antibody (cross-reactivity rat I C-peptide $30 \%$, rat II C-peptide 100\%) as in the insulin assay. The sensitivity and range were 50-1,600 pM. The intra- and interassay variation for C-peptide was 4.0 (low) and 7.7 (high), and 10.1 (low) and $7.1 \%$ (high), respectively.

Liver enzymes were measured in triplicate $(23)$ by a fluorimetric method using a spectrophotometer (Cobas Mira, Roche, Montclair, NJ). Oxygen, carbon dioxide, potassium, and $\mathrm{pH}$ were measured immediately with a gas meter (BGElectrolytes, Instrumentation Laboratory, Inc., Lexington, MA). 
Table III. Results of Pulse Detection Analyses in Isolated Perfused Livers

\begin{tabular}{|c|c|c|c|c|c|c|}
\hline Days $T x$ & $\begin{array}{l}\text { Number } \\
\text { of rats }\end{array}$ & $\begin{array}{l}\text { Number } \\
\text { showing } \\
\text { pulsatility }\end{array}$ & $\begin{array}{l}\text { Nonpulsatile } \\
\text { insulin } \\
\text { secretion* }\end{array}$ & $\begin{array}{c}\text { Pulse } \\
\text { frequency }\end{array}$ & $\begin{array}{c}\text { Pulse } \\
\text { increment }^{\ddagger}\end{array}$ & $\begin{array}{l}\text { Glucagon } \\
\text { response* }\end{array}$ \\
\hline & & & nmol/min & pulses/h & nmol/min & nmols $\min$ \\
\hline 2 & 5 & 1 & $2.7 \pm 0.8$ & $4.5^{8}$ & $5.9^{\S}$ & $40.8 \pm 9.9$ \\
\hline 7 & 5 & 0 & $1.1 \pm 0.3$ & - & - & $31.7 \pm 9.9$ \\
\hline 30 & 5 & 1 & $1.4 \pm 0.3$ & $3.0^{8}$ & $7.4^{8}$ & $36.7 \pm 8.4$ \\
\hline 200 & 12 & 12 & $10.5 \pm 3.0^{\prime \prime}$ & $3.9 \pm 0.3$ & $15.2 \pm 2.4^{1}$ & $149.3 \pm 40.0^{\|}$ \\
\hline 200 before barbiturate & 4 & 4 & $12.9 \pm 3.5$ & $4.1 \pm 0.3$ & $17.6 \pm 4.5$ & - \\
\hline 200 after barbiturate & 4 & 4 & $14.1 \pm 3.3$ & $4.0 \pm 0.7$ & $20.0 \pm 4.2$ & - \\
\hline
\end{tabular}

${ }^{8}$ Only the cases showing pulses included. * Tested by Newman-Keul's test for multiple comparisons. ${ }^{\ddagger}$ Tested by Student's nonpaired $t$ test. " $200>2,7$, and $30(P<0.01),{ }^{\prime} 200>2+30(P<0.05)$. When comparing pulse frequency and pulse amplitude, days 2 and 30 were considered one group because of the small numbers.

\section{Pulse detection}

Statistically significant insulin secretory pulses were determined using a peak detection algorithm, cluster analysis (24). The insulin delivery rates from isolated perfused livers given a constant insulin infusion (to define model noise) and pulsatile insulin delivery (to insure detection as true positive pulse events) are illustrated in Fig. 1. During the constant insulin infusion ( $0-40 \mathrm{~min}$ ), the rates of insulin delivery to (perfusate insulin concentration $\times$ buffer flow rate) and from (effluent insulin concentration $\times$ buffer flow rate) the liver were relatively stable (coefficients of variations 6.7 and $8.3 \%$ ). During pulsatile insulin delivery (40-80 min), distinct insulin pulses were delivered to and obtained from the isolated perfused livers. Deconvolution analysis was not neces-

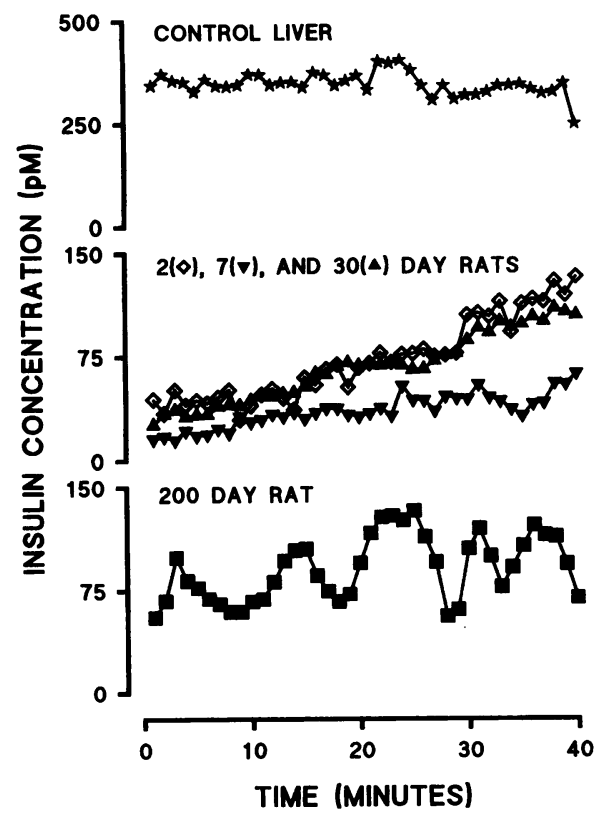

Figure 3. The assay and model noise are illustrated in the top panel which shows the effluent insulin concentration from a control liver perfused with insulin at a constant rate $(1.0 \mathrm{mU} / \mathrm{min})$. In the middle panel, representative insulin concentration profiles are shown from early transplants versus a 200-d transplant in the bottom panel (concentrations shown as assayed after 1:10 dilution in 200-d Tx and undiluted in early Tx). Note that the magnitude of the pulses in the 200-d Tx is striking in comparison to the model/assay noise. sary in analyzing these secretion data, since buffer flow $\times$ concentration equals the absolute rate of insulin secretion. Accordingly, statistical identification of increases and decreases in insulin secretory rate was carried out by cluster analysis, a discrete peak-detection method (24). To minimize false-positive errors because of the erroneous identification of random experimental variations as apparent insulin secretory peaks, the insulin release rates from the three livers perfused with insulin at a constant rate (Fig. 1, $\mathrm{t}=0-40 \mathrm{~min}$ ) were subjected to cluster analysis. The detection threshold ( $t$ statistics for significant upstrokes and downstrokes in the data) and the degree of stringency (number of points in the test nadirs and peaks) were varied from 0.5 to 8.0 and from 1 to 5 , respectively. Optimal peak detection was defined as a false-positive rate of $\leq 1 \%$ (no more than one peak detected per 100 observations in the constant infusion protocol), with $100 \%$ detection of the pre- and posthepatic true-positive insulin pulses. Cluster parameters satisfying these conditions consisted of three samples in both the test nadir and peak and had $t$ statistics of 4.0 for significant increases and decreases in the insulin secretory rates. The pooled $t$ statistics were applied to a dose-dependent variance model calculated from all the sample observations in each insulin secretory profile, such that the within-sample variance was determined as the best-fit power function of the sample mean (25). Under these conditions all 12 infused and posthepatic insulin peaks were detected, and only one false-positive error was incurred in analyzing all three constant infusion series. Measurements of interest included: $(a)$ number of statistically significant insulin secretory pulses, each of which consisted of a significant increase in insulin secretory rate above preceding nonpulsatile secretion rate (or nadir), followed by a statistically significant decrease in insulin secretory rate; $(b)$ nonpulsatile insulin secretory rate or the interpeak valley (nadir) secretory rate; and $(c)$ insulin secretory pulse amplitude or the incremental increase in the insulin secretory rate in the peak compared with the preceding nadir.

The experimental design used (constant hyperglycemia) resulted in a second-phase insulin response during the sampling period (the firstphase response having been during the presampling period). If this second-phase response was complete during the sampling period (i.e., secretion rate returned to or near to baseline), it was detected by cluster analysis as a single pulse of $\sim 30$-min duration. To distinguish this second-phase response from high frequency pulsatile insulin secretion, we considered transplanted islets as showing pulsatility only if more than one pulse was detected during the sampling period.

\section{Statistics}

Data in the text and figures are given as mean \pm SEM. Statistical significance was considered to be $P<0.05$. Newman-Keul's test for multiple comparisons was used when comparing the four groups (i.e., 2, 7, 30, and $200 \mathrm{~d}$ ). Otherwise, specific statistical methods used are described in Methods and Results. 


\section{Results}

The mean perfusate insulin concentrations for each group in relation to the assay range are shown in Fig. 2. Of the 1,470 samples assayed for insulin in these studies, 7 were at or slightly below the linear portion of the binding curve for this assay (4 of these were the first 4 samples of one 7-d Tx), and 13 out of 15 of the early transplant livers had no samples at or below the linear portion of the assay binding curve. To avoid sample concentrations exceeding the linear portion of the assay binding curve, the samples in 10 out of 12 of the $200-d, 2$ out of 5 of the 2-d, 0 out of 5 of the 7-d, and 1 out of 5 of the 30-d Tx were diluted. In 12 out of 12 of the chronically transplanted livers (200-d Tx), insulin was secreted in clearly detectable coordinate pulses; the mean frequency was $3.9 \pm 0.3$ pulses $/ \mathrm{h}$ (periodicity $15.4 \pm 1.2 \mathrm{~min}$ ), mean pulse duration was $10.9 \pm 0.8$ min, and the mean amplitude was $15.2 \pm 2.4 \mathrm{nmol} / \mathrm{min}$. In contrast, only one out of five of the 2-d, zero out of five of the 7-d, and one out of five of the 30-d Tx livers showed high frequency pulsatile insulin secretion (Table III). Representative insulin concentration profiles from each group are shown in Fig. 3, demonstrating the clear insulin concentration pulses in the 200$\mathrm{d}$ but not the early $\mathrm{Tx}$ livers. Note that the insulin concentrations (after dilution) in the 200-d perfusate were comparable with those in the early livers so that the absence of pulses in the latter was not an assay artifact. Representative insulin secretion (concentration $\times$ buffer flow) profiles for 2-, 7-, 30-, and 200$\mathrm{d}$ transplanted livers are shown in Fig. $4, A$ and $B$, and the means of all livers are shown in Fig. 5.

From Fig. 5, it is clear that the total insulin secretion rates in response to an identical stimulus (i.e., $10 \mathrm{mM}$ glucose) increased markedly by $200 \mathrm{~d}$ of Tx versus $2-, 7-$, or $30-\mathrm{d}$ Tx ( $P$ $<0.01, P<0.001$, and $P<0.001$ ) (Table III). Since relatively few early (one 2-d and one 30-d) Tx showed pulsatile insulin secretion, to compare the effects of duration of transplant on the pattern of pulsatile insulin secretion we considered the 2-, 7-, and 30-d Tx together (i.e., early Tx) versus $200-d$ Tx (i.e., late $\mathrm{Tx}$ ). In the livers that showed pulsatile insulin secretion, the pulse increment of insulin secretory bursts was higher (15.2 \pm 2.4 vs $6.7 \pm 0.8 \mathrm{nmol} / \mathrm{min}, P<0.05)$, but the frequency did not differ in the late $(3.9 \pm 0.2$ pulses $/ h)$ versus early (3.8 \pm 0.8 pulses $/ \mathrm{h}$ ) Tx (Table III). Both the nonpulsatile and total insulin secretion rates were also greater in the late versus early Tx livers $(P<0.001$, Table III). In the livers exhibiting pulsatile insulin secretion, there was a clear relationship between the rate of nonpulsatile insulin secretion and the amplitude of coordinate insulin secretory bursts (Fig. $6 ; r=0.92, P$ $<0.001)$. It is noteworthy that an insulin response to a glucagon bolus was present in all livers tested, with the magnitude of this first-phase response being greater in the late versus early $\mathrm{Tx}$ livers $(P<0.001$; Table III $)$.

Since C-peptide is not cleared by the liver, in five rats both insulin and C-peptide concentrations were measured in perfusate samples to exclude the possibility that oscillations in insulin concentration were due to oscillations in insulin clearance (Fig. 7). Cross-correlation analysis between insulin and C-peptide concentrations revealed a high degree of correlation at zero lag (i.e., insulin and C-peptide peaks are simultaneous, $P$ $<0.0001)$. These data confirmed that pulsatile insulin efflux from the perfused livers was due to coordinate pulsatile secretion rather than pulsatile hepatic insulin clearance. It was also plausible that apparent pulses of insulin secretion were due to
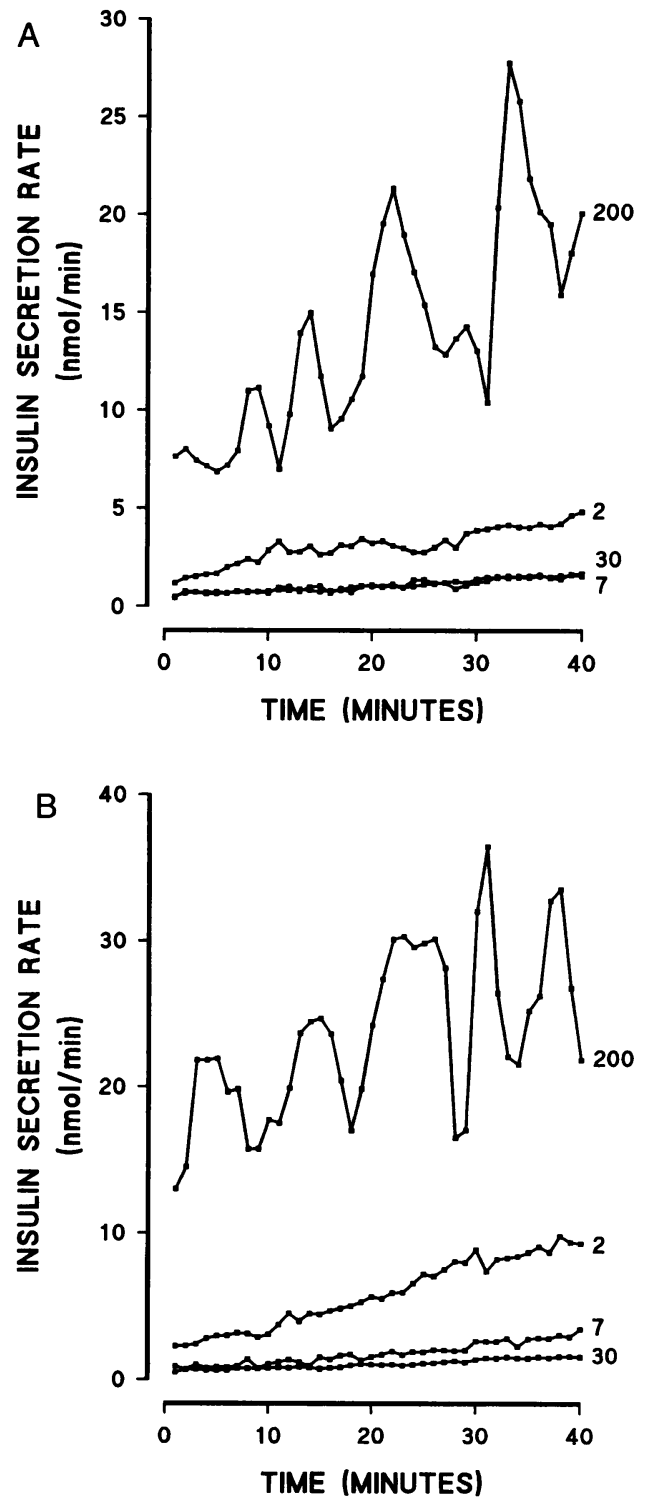

Figure 4. ( $A$ and $B$ ) Insulin secretion rates (nanomoles per minute) from eight representative livers transplanted $2,7,30$, and $200 \mathrm{~d}$ previously during a constant perfusion of Kreb's buffer with a glucose concentration of $10 \mathrm{mM}$

intermittent changes in hepatic blood flow. However, crosscorrelation analysis between hepatic flow rates and simultaneous insulin concentrations indicated a negative correlation (mean $z$ score of $-1.5 \pm 0.05, P<0.001$ versus nonrandom associations by the Kolmogorov-Smirnov statistic), indicating that any fluctuations in perfusate flow rates were not responsible for periodic increases in insulin secretion.

A perfusate concentration of $25 \mu \mathrm{g} / \mathrm{ml}$ of pentobarbital had no effect on the total $(13.9 \pm 3.9$ vs $15.9 \pm 3.9 \mathrm{nmol} / \mathrm{min})$, nonpulsatile $(12.9 \pm 3.5 \mathrm{vs} 14.1 \pm 3.3 \mathrm{nmol} / \mathrm{min}$ ), or pulsatile (pulse increment $17.6 \pm 4.5$ vs $20.0 \pm 4.2 \mathrm{nmol} / \mathrm{min}$, pulse frequency $4.1 \pm 0.3$ vs $4.0 \pm 0.7$ pulses $/ h$ ) insulin secretion rates (Fig. 8 and Table III). Since this dose of pentobarbital causes anesthesia in a rat, these data suggest that the intrahepatic pacemaker and system for propagation of the signal between islets are relatively resistant to suppression by anesthetic doses of barbiturate and 


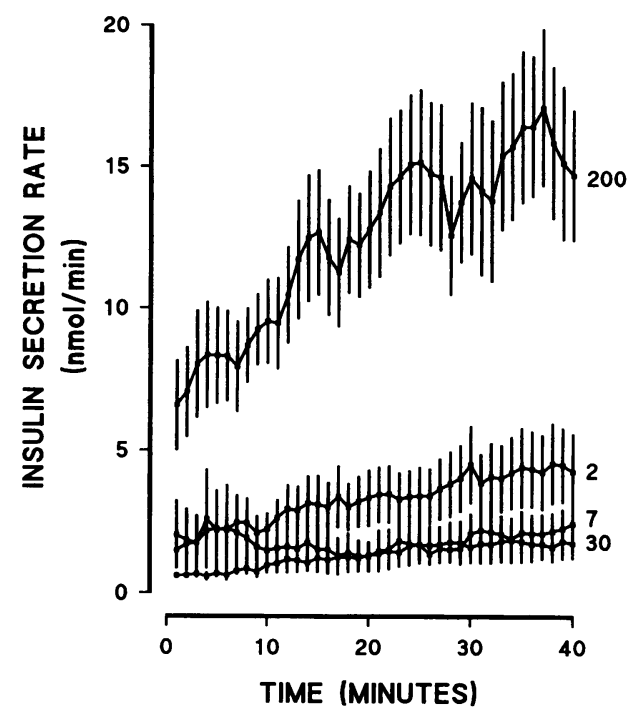

Figure 5. Mean insulin secretion rates (nanomoles per minute) from islets transplanted $2(n=5), 7(n=5), 30(n=5)$, and $200(n=12)$ d previously during a constant perfusion of Kreb's buffer with a glucose concentration of $10 \mathrm{mM}$.

therefore are analogous to the sinoatrial node and Purkinje tissue present in the heart (18).

Using synaptophysin labeling, islet innervation was clearly demonstrable in 12 out of 12200 -d Tx livers but not in any of the early Tx livers, consistent with previous studies (12-14). Innervation was confirmed by the presence of synaptophysin staining nerve endings penetrating the transplanted islet as indicated in Fig. 9. Nerve endings stained by synaptophysin were present in the periportal areas in early transplanted islets, but fibers were not found penetrating the transplanted islets.

\section{Discussion}

It is well established that islets distributed within the pancreatic parenchyma secrete insulin in high frequency coordinate secretory bursts (10). In the present study, we sought to determine if these coordinate high frequency secretory bursts are present when islets are transplanted via the portal vein and thereby distributed to the liver. We report here that early after transplantation these coordinate high frequency secretory bursts are absent, but that they are reestablished by $200 \mathrm{~d}$ after transplantation.

Since single islets secrete insulin in a pulsatile manner within hours of isolation (26), it is likely that the early transplanted islets in the present studies secreted insulin individually in a pulsatile manner. However, since the net insulin output from the liver (the sum of all islets) was not pulsatile in the early transplanted livers, it appears that the mechanism for interislet communication that leads to coordinate pulsatile insulin secretion was not present during the first $30 \mathrm{~d}$ after transplantation. Intrahepatic transplanted islets become vascularized within $7 \mathrm{~d}$ of transplantation (12). The present data therefore appear to lend additional argument against the hypothesis that coordinate pulsatile insulin secretion in vivo is due to entrainment of islets by either oscillations in circulating glucose (8) or an intrinsic metabolic cycle present in pancreatic islets (26). It has been suggested that coordinate secretory burst activity is achieved through the intrapancreatic nerve network $(10,27)$. There is indeed an intrinsic intrapancreatic neural network that does not degenerate after central denervation (28) and which is intimately associated with islets as demonstrated by the nerve fibers entering each islet (28). Furthermore, this intrapancreatic neural network has well defined intrapancreatic ganglia, which have been shown to have spontaneous pacemaker activity (29). If coordinate insulin secretory burst activity from widely dispersed islets depends on interislet communication through intrinsic organ nerve fibers, this coordinate pulsatility should be absent if interislet communication is disrupted, such as would occur immediately after isolation and transplantation of islets into the liver. In these studies, only one out of five livers showed high frequency pulsatility at $2 \mathrm{~d}$ after transplantation. Since islets at this time are still intraportal where they may be aggregated (12), this single case most likely represented coordinate pulsatile insulin secretion from an aggregate of islets as occurs in periperfused islets $(30,31)$. However, by $7 \mathrm{~d}$ after transplantation the islets had become topographically dispersed within the hepatic parenchyma (12), and at that time there were no high frequency coordinate secretory bursts present, although they became reestablished between 30 and $200 \mathrm{~d}$, which coincides with the time period described for the establishment of islet reinnervation (12-14). Taken together these data are consistent with the hypothesis that coordinate high frequency insulin secretory bursts by islets dispersed within an organ are mediated by interislet innervation, which allows for transmission of a synchronizing signal or a wave of depolarization throughout the islet population (27). However, these data raise several questions.

First, what is the pacemaker for such a wave of depolarization? Since only islet tissue is transplanted and islets have independent pacemaker function, it is possible that one or more of the islets directly serves as a pacemaker. Alternatively, there may be intrahepatic ganglia that are similar to those described in the pancreas (29) that serve as a pacemaker. Indeed, it is perhaps more plausible that reinnervation of intrahepatic islets would result in coordinate spontaneous depolarization from an intrinsic hepatic pacemaker than through a mechanism that required direct islet to islet interconnection. It is of note that the frequency of insulin secretory bursts by islets transplanted in

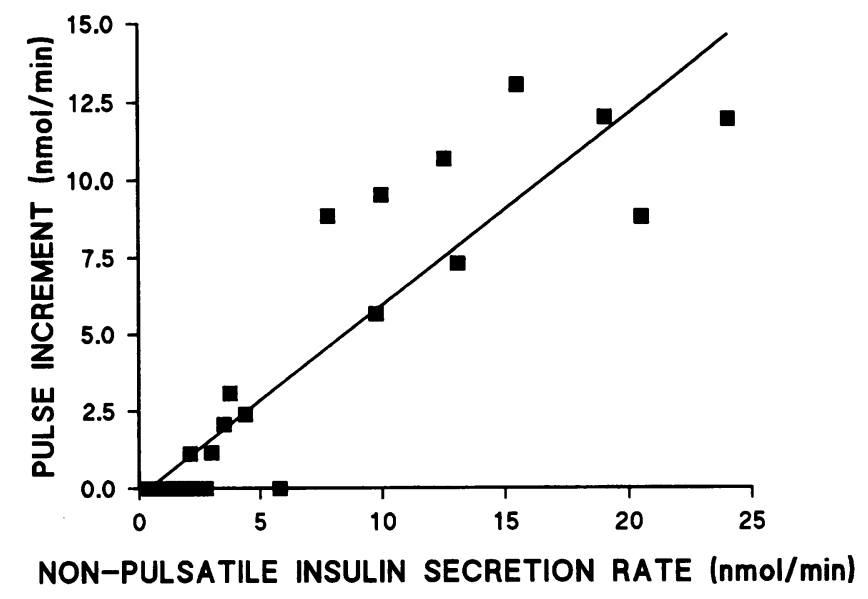

Figure 6. Relationship between the mean pulse amplitude (above nonpulsatile) and nonpulsatile insulin secretion rate for each liver studied showing a highly significant positive correlation $(r=0.92, P<0.01)$. 


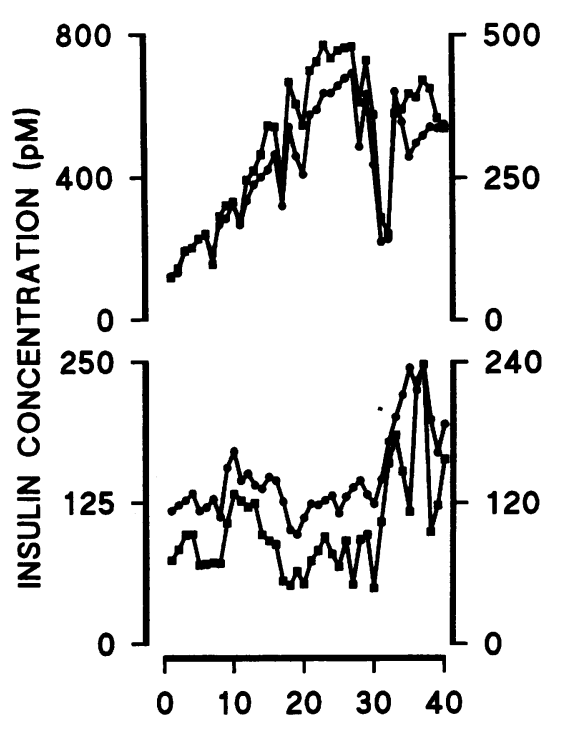

TIME (MINUTES)

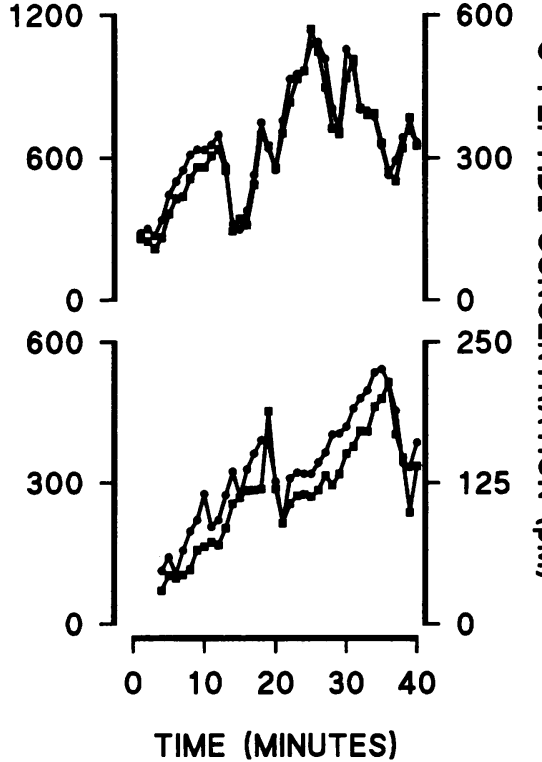

Figure 7. Simultaneous insulin (left) (picomolars) and C-peptide concentrations (right) (picomolars) in perfusate collected from livers with islets transplanted $200 \mathrm{~d}$ previously. Samples were collected during a constant perfusion of Kreb's buffer with a glucose concentration of $10 \mathrm{mM}$ and show concordant insulin and C-peptide oscillations. the isolated perfused liver is slower (periodicity $\sim 14 \mathrm{~min}$ ) than that seen in the isolated perfused pancreas ( $\sim 5 \min [32])$, which may indicate a different pacemaker. On the other hand, the frequency of insulin secretory bursts by the isolated livers is comparable with that seen in vivo in rats (periodicity $\sim 15$ $\min [33]$ ).

A second question provoked by these and other data is what is the significance of interislet communication and coordinate pulsatile insulin secretion? With respect to the clinical relevance of coordinate insulin secretory burst activity, it has been suggested that pulsatile insulin delivery enhances insulin action in target tissues (34). However, the impact of pulsatile versus constant insulin secretion by intrahepatic islets on insulin sensitivity has not yet been determined. Coordinate interislet communication is also of potential importance in the amplification of

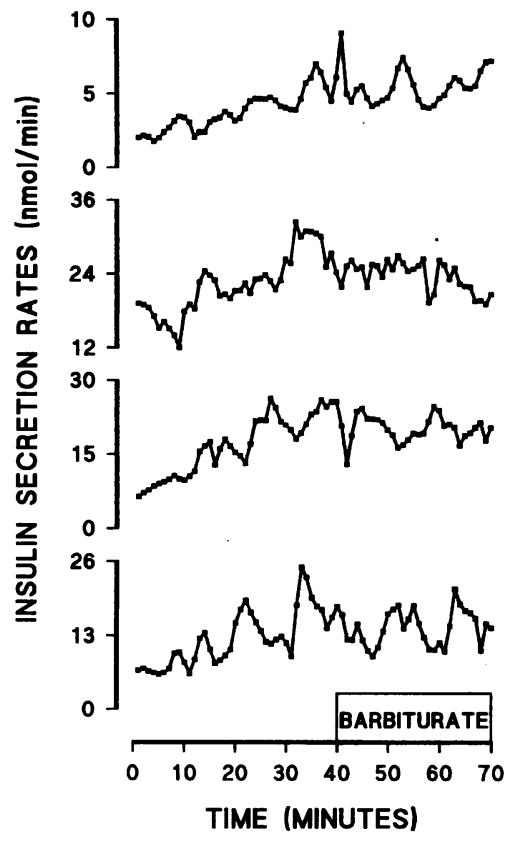

Figure 8. Insulin secretion rates (nanomoles per minute) from islets transplanted $200 \mathrm{~d}$ previously in four livers during a constant perfusion of Kreb's buffer with glucose $10 \mathrm{mM}$ before $(0$ $40 \mathrm{~min}$ ) and after (41$70 \mathrm{~min}$ ) perfusion with pentobarbital, $25 \mu \mathrm{g} / \mathrm{ml}$. There were no changes in pulsatile insulin secretion in response to the pentobarbital. the insulin secretion response to secretagogues. When a borderline number of islets are transplanted, long-term islet viability depends upon exogenous insulin treatment for several weeks (35). This implies that it takes several weeks for transplanted islets to achieve their maximal potential rates of insulin secretion. It is not clear what mechanisms are involved in this delay. One possibility is that this delay represents the time taken to reestablish coordinate pulsatile insulin secretion. Since aggregated $\beta$-cells secrete more insulin than isolated $\beta$-cells in response to a glucose challenge (36), it is plausible that interislet communication also results in enhanced insulin secretion per islet. In the present studies the magnitude of the insulin secretory response was greater in the 200 -d versus early transplanted islets with exposure to an identical secretagogue challenge ( 10 $\mathrm{mM}$ glucose). However, it is also likely that islet hyperplasia in response to prolonged transplantation in aging, obese rats may have contributed to the increased rate of insulin secretion in the 200-d transplanted rats.

In this study we also sought to determine if the coordinate insulin secretory bursts were disrupted by an anesthetic dose of the barbiturate pentobarbital. The failure of an anesthetic dose of pentobarbital to inhibit pulsatile insulin secretion in these studies is consistent with the hypothesis that coordinate interislet communication between intrahepatic islets is achieved by a pacemaker and neural system analogous to the sinoatrial node and Purkinje tissue present in the heart.

In conclusion, coordinate pulsatile insulin delivery was reproducibly detected in the effluent from isolated livers transplanted with pancreatic islets $200 \mathrm{~d}$ previously. This pulsatile mode of insulin secretion was not due to changes in perfusate flow or hepatic insulin clearance and therefore reflected coordinate pulsatile insulin secretion by transplanted islets. The establishment of coordinate pulsatile insulin secretion in intrahepatic islets paralleled histological reinnervation of islets, suggesting, but not proving, that intrahepatic reinnervation may have been of importance in the establishment of coordinate pulsatile insulin secretion. These data also imply that fully established engraftment of intrahepatic transplanted islets is more complex and takes longer than simple vascularization. 

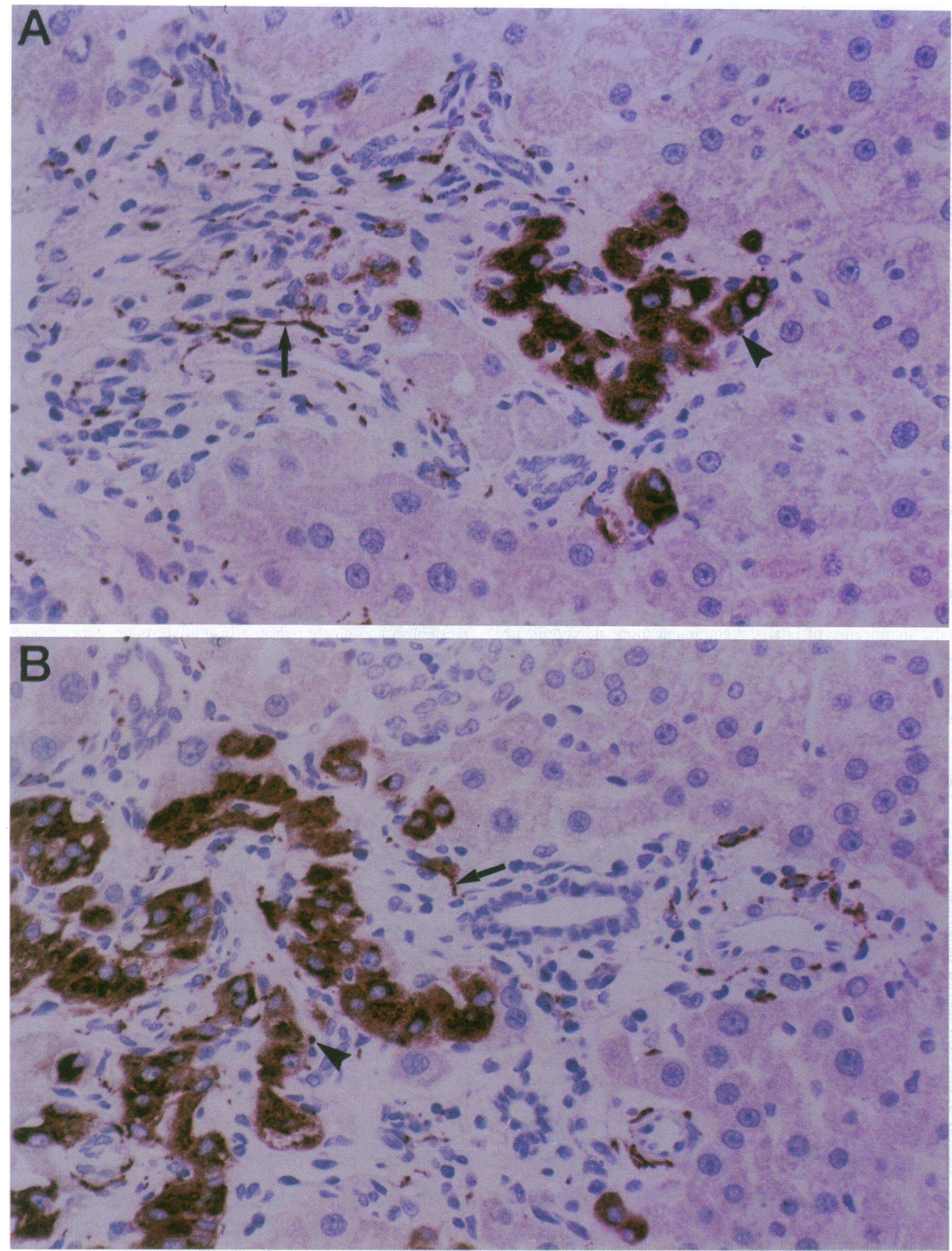

Figure 9. ( $A$ and $B$ ) Sections from livers (with 200-d transplanted islets) stained by immunohistochemistry for synaptophysin are shown. Both islets (one is shown in each section) and nerve tissue are stained for synaptophysin. Axon fibers are shown clearly in longitudinal section (full arrows) and in section (arrowheads) penetrating the islets. 


\section{Acknowledgments}

We acknowledge the excellent technical assistance of Jane Kahl, Karen Laakso, Jane Bailey, and Diane Roddy.

The present study was supported by U.S. Public Health Service grants DK-44341 and RR-00585 and the Mayo Foundation. Dr. Niels Pørksen was supported by the University of Aarhus, Denmark. Dr. Timothy D. O'Brien was supported by Pfizer Pharmaceutical.

\section{References}

1. Federlin, K. F., R. G. Bretzel, and B. J. Hering. 1992. Islet transplant registry. In Pancreatic Islet Cell Transplantation. C. Ricardi, editor. R. G. Langes Co., Austin. 462-472.

2. Lacy, P. E. 1992. Islet transplantation: the future. In Pancreatic Islet Cell Transplantation. C. Ricardi, editor. R. G. Langes Co., Austin. 394-399.

3. Scharp, D. W., P. E. Lacy, J. V. Santiago, C. S. McCullough, L. G. Weide, P. J. Boyle, L. Falqui, P. Marchetti, C. Ricordi, R. L. Gingerich, et al. 1991. Results of our first nine intraportal islet allografts in Type 1, insulin-dependent diabetic patients. Transplantation (Baltimore). 51:76-85.

4. Warnock, G. L., N. M. Kneteman, E. A. Ryan, A. Rabinovitch, and R. V. Rajotte. 1992. Long-term follow-up after transplantation of insulin-producing pancreatic islets into patients with Type 1 (insulin-dependent) diabetes mellitus. Diabetologia. 35:89-95.

5. Pyzdrowski, K. L., D. M. Kendall, J. B. Halter, R. E. Nakhler, D. E. R. Sutherland, and R. P. Robertson. 1992. Preserved insulin secretion and insulin independence in recipients of islet autografts. N. Engl. J. Med. 327:220-226.

6. Lang, D. A., D. R. Matthews, D. Phil, J. Peto, and R. C. Turner. 1979. Cyclic oscillations of basal plasma glucose and insulin concentrations in human beings. N. Engl. J. Med. 301:1023-1027.

7. Polonsky, K. S., B. D. Given, and E. Van Cauter. 1988. Twenty-four-hour profiles and pulsatile patterns of insulin secretion in normal and obese subjects. J. Clin. Invest. 81:442-448.

8. Sturis, J., E. Van Cauter, J. D. Blackman, and K. S. Polonsky. 1991. Entrainment of pulsatile insulin secretion by oscillatory glucose infusion. J. Clin. Invest. 87:439-445.

9. Shapiro, E. T., S. Strasser, K. S. Polonsky, R. Alejandro, and D. H. Mintz. 1991. Persistence of oscillatory insulin secretion in denervated islet cell autografts. Transplantation (Baltimore). 52:574-576.

10. Stagner, J. I., E. Samols, and G. C. Weir. 1980. Sustained oscillations of insulin, glucagon, and somatostatin from the isolated canine pancreas during exposure to a constant glucose concentration. J. Clin. Invest. 65:939-942.

11. Miller, R. E. 1981. Pancreatic neuroendocrinology: peripheral neural mechanisms in the regulation of the islets of Langerhans. Endocr. Rev. 2:471494.

12. Griffith, R. C., D. W. Scharp, B. K. Hartman, W. F. Ballinger, and P. E. Lacy. 1977. A morphologic study of intrahepatic portal-vein islet isografts. Diabetes. 26:201-214.

13. Cossel, L., F. Wohlrab, W. Blech, and H. J. Hahn. 1990. Morphological findings in the liver of diabetic rats after intraportal transplantation of neonatal isologous pancreatic islets. Virchows Arch. B Cell Pathol. 59:65-77.

14. Korsgren, O., L. Jansson, A. Andersson, and F. Sundler. 1993. Reinnervation of transplanted pancreatic islets. Transplantation (Baltimore). 56:138-143.

15. Aynsley-Green, A., J. F. Biebuyck, and K. G. M. M. Alberti. 1973. Anaesthesia and insulin secretion: the effects of diethyl ether, halothane, pentobar- bitone sodium and ketamine hydrochloride on intravenous glucose tolerance and insulin secretion in the rat. Diabetologia. 9:274-281.

16. Vanhelder, W. P. 1981. Modification of pulsatile pattern of basal insulin secretion in the dog by general anesthesia (Nembutal). Experientia (Basel). 37:1032-1033.

17. Hemelrijck, J. V., J. M. Gonzales, and P. F. White. 1993. Pharmacology of intravenous anesthetic agents. In Principles and Practice of Anesthesiology. M. C. Rogers, B. G. Covino, and D. E. Longnecker, editors. Mosby-Year Book, Inc., St. Louis. 1134-1137.

18. Todd, M. M., J. C. Drummond, and H.S.U. 1984. The hemodynamic consequences of high-dose methohexital anesthesia in humans. Anesthesiology. 61:495-501.

19. Munn, S. R., D. B. Kaufman, M. J. Field, A. B. Viste, and D. E. R Sutherland. 1989. Cold-storage preservation of the canine and rat pancreas prior to islet isolation. Transplantation (Baltimore). 47:28-31.

20. Wolkoff, A. W., K. L. Johansen, and T. Goeser. 1987. The isolated perfused rat liver: preparation and application. Anal. Biochem. 167:1-14.

21. Gores, G. J., L. J. Kost, and N. F. LaRusso. 1986. The isolated perfused rat liver: conceptual and practical considerations. Hepatology. 6:511-517.

22. Sugano, T., K. Suda, M. Shimada, and N. Oshino. 1978. Biochemical and ultrastructural evaluation of isolated rat liver systems perfused with a hemoglobinfree medium. J. Biochem. 83:995-1007.

23. Gay, R. J., R. B. McComb, and G. N. Bowers, Jr. 1968. Optimum reaction conditions for human lactate dehydrogenase isoenzymes as they affect total lactate dehydrogenase activity. Clin. Chem. 14:740-752.

24. Veldhuis, J. D., and M. L. Johnson. 1986. Cluster analysis: a simple, versatile and robust algorithm for endocrine pulse detection. Am. J. Physiol. 250:E486-E493.

25. Urban, R. J., W. S. Evans, A. D. Rogol, D. L. Kaiser, M. L. Johnson, and J. D. Veldhuis. 1988. Contemporary aspects of discrete peak detection algorithms. I. The paradigm of the luteinizing hormone pulse signal in men. Endocr. Rev. 9:3-37.

26. Hayek, A. 1981. Preservation of insulin release periodicity from individual islets of obese Zucker rats. Horm. Metab. Res. 13:119-120.

27. Stagner, J. I., and E. Samols. 1985. Role of intrapancreatic ganglia in regulation of periodic insular secretions. Am. J. Physiol. 248 (Endocrinol. Metab. 11):E522-E530.

28. Honjin, R. 1956. The innervation of the pancreas of the mouse, with special reference to the structure of the peripheral extension of the vegetative nervous system. J. Comp. Neurol. 104:331-361.

29. King, B. F., J. A. Love, and J. H. Szurszewski. 1989. Intracellular recordings from pancreatic ganglia of the cat. J. Physiol. 419:379-403.

30. Bergstrom, R. W., W. Y. Fujimoto, D. C. Teller, and C. De Haën. 1989. Oscillatory insulin secretion in perifused isolated rat islets. Am. J. Physiol. 257 (Endocrinol. Metab. 20):E479-E485.

31. Chou, H.-F., and E. Ipp. 1990. Pulsatile insulin secretion in isolated rat islets. Diabetes. 39:112-117.

32. Stagner, J. I., and E. Samols. 1988. Comparison of insulin and glucagon pulsatile secretion between the rat and dog pancreas in vitro. Life Sci. 43:929934.

33. Chou, H.-F., R. McGivern, N. Berman, and E. Ipp. 1991. Oscillations of circulating plasma insulin concentrations in the rat. Life Sci. 48:1463-1469.

34. Matthews, D. R., B. A. Naylor, R. G. Jones, G. M. Ward, and R. C Turner. 1983. Pulsatile insulin has greater hypoglycemic effect than continuous delivery. Diabetes. 32:617-621.

35. Hayek, A., A. D. Lopez, and G. M. Beattie. 1988. Decrease in the number of neonatal islets required for successful transplantation by strict metabolic control of diabetic rats. Transplantation (Baltimore). 45:940-942.

36. Bosco, D., and P. Meda. 1991. Actively synthesizing $\beta$-cells secrete preferentially after glucose stimulation. Endocrinology. 129:3157-3166. 